\title{
Pompéi. Villa de Diomède
}

Campagne d'étude 2014

Hélène Dessales, Jean Ponce, Mathilde Carrive, Julien Cavero, Julien Dubouloz, Éloïse Letellier, Frédérique Marchand- Beaulieu, Florence Monier, Ambre Péron, Agnès Tricoche et Yves Ubelmann

\section{OpenEdition}

12 Journals

Édition électronique

URL : http://journals.openedition.org/cefr/1293

DOI : $10.4000 /$ cefr. 1293

ISSN : 2282-5703

Éditeur

École française de Rome

Référence électronique

Hélène Dessales, Jean Ponce, Mathilde Carrive, Julien Cavero, Julien Dubouloz, Éloïse Letellier, Frédérique Marchand- Beaulieu, Florence Monier, Ambre Péron, Agnès Tricoche et Yves Ubelmann, « Pompéi. Villa de Diomède », Chronique des activités archéologiques de l'École française de Rome [En ligne], Les cités vésuviennes, mis en ligne le 02 février 2015, consulté le 12 septembre 2019. URL http://journals.openedition.org/cefr/1293; DOI : 10.4000/cefr.1293

Ce document a été généré automatiquement le 12 septembre 2019

(c) École française de Rome 


\title{
Pompéi. Villa de Diomède
}

\author{
Campagne d'étude 2014 \\ Hélène Dessales, Jean Ponce, Mathilde Carrive, Julien Cavero, Julien \\ Dubouloz, Éloïse Letellier, Frédérique Marchand- Beaulieu, Florence \\ Monier, Ambre Péron, Agnès Tricoche et Yves Ubelmann
}

\section{NOTE DE L'ÉDITEUR}

Hélène Dessales (ENS, UMR 8546 / PSL - IUF) assure la coordination scientifique, Jean Ponce (ENS, UMR 8548 / PSL, Inria - IUF) la coordination de la recherche informatique. Julien Cavero (Labex TransferS) a en charge le système d'information géographique, Agnès Tricoche (Labex TransferS) la gestion des bases de données, Julien Dubouloz (Université d'Aix-Marseille, UMR 7297) l'étude du parcellaire et de la propriété, Mathilde Carrive (EFR) et Florence Monier (CNRS, UMR 8546) l'étude des enduits peints, Frédérique Marchand-Beaulieu (CNRS, UMR 8546) l'étude des pavements, Éloise Letellier (EFR) l'étude stratigraphique des élévations, Ambre Péron (ENS) l'étude des graffitis historiques et Yves Ubelmann (Iconem) la modélisation 3D.

\section{NOTE DE L'AUTEUR}

Nous exprimons toute notre reconnaissance aux institutions et aux personnes qui ont soutenu ce projet et en ont permis la réalisation. Nos vifs remerciements vont à Massimo Osanna, Surintendant, ainsi qu'à Grete Stefani, Directrice des fouilles de Pompéi, pour leur soutien constant et leur collaboration au cours de nos recherches. Nous avons le plaisir de remercier Fabio Galeandro, responsable archéologique en charge du secteur, Vincenzo Sabini, assistant scientifique, pour le suivi attentif de toute la mission sur le terrain. Nous remercions enfin tous les étudiants qui ont participé à la campagne de terrain, à différents degrés de leur parcours et de leur formation (archéologie, géologie, architecture et ingénierie) : Yacine Benjelloun (ENS, doctorant en géologie) ; Jeanne Capelle (ENS, doctorante en archéologie) ; Antonella Ciotola (Università degli Studi di 
Napoli Federico II, doctorante en archéologie) ; Ségolène Delamare (Université de Paris 1, Master 2 d'archéologie) ; Alexandre Gouverneur (ENS, Master 2 d'archéologie) ; Stefano Iavarone (Università degli Studi di Napoli «L'Orientale », doctorant en archéologie) ; Sophie Mieczkowski (ENS, Master 1 d'archéologie) ; Simona Troisi (Università degli Studi di Salerno, Laurea magistrale in Archeologia e Culture Antiche) ; Marianna Vigorito (Università degli Studi di Salerno, Scuola di Specializzazione in Beni archeologici) ; Stefano D'Arco et Gabriele Pepe (étudiants architectes, Università degli Studi di Napoli Federico II) ; Giuseppina De Martino (doctorante en ingénierie, Università degli Studi di Napoli Federico II).

1 Dans la continuité du programme initié en $2013^{1}$, l'objectif de la deuxième campagne menée sur la Villa de Diomède était de mettre en évidence la chronologie du bâtiment et son rapport aux espaces environnants. Pour ce faire, l'étude archéologique a été centrée sur les façades est et sud de la villa, afin d'éclaircir le rapport à la rue (via delle Tombe) et aux tombes adjacentes. Il convenait d'explorer davantage l'évolution de la villa en fonction des rapports de propriété et d'occupation de l'espace qui organisent ce secteur périurbain de Pompéi.

Cinq secteurs ont pour cela été distingués : sur la façade est, les secteurs 100 et 101 ; sur la façade sud, les secteurs 102, 103, 104 et 105 (fig. 1). Ils ont été l'objet d'une analyse stratigraphique et de relevés systématiques, réalisés du 5 au 24 mai 2014.

Fig. 1 - Plan du rez-de-chaussée de la Villa de Diomède et des environs du bâtiment.

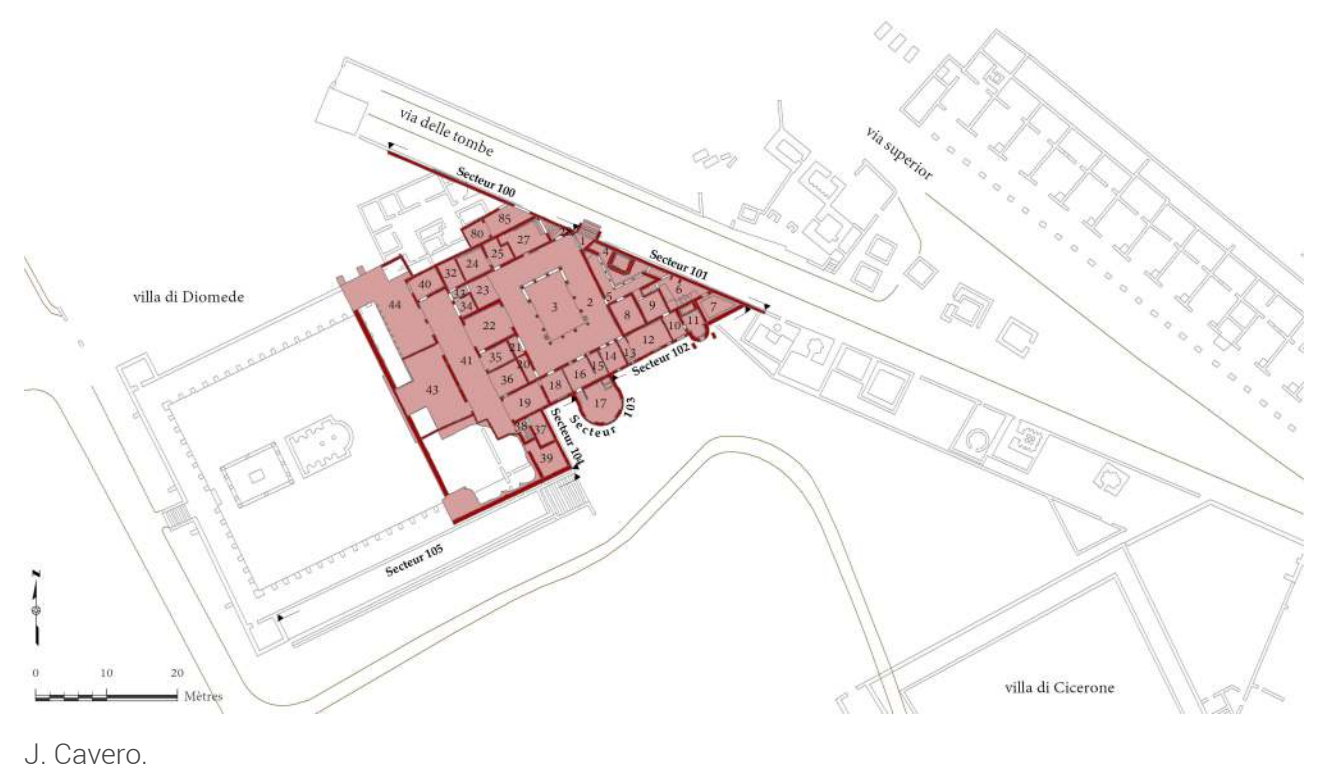

3 Cette campagne de terrain a associé un stage de formation à l'archéologie de la construction romaine pour des étudiants français et italiens inscrits en master et en doctorat. Ce stage a été intégré au programme du nouveau Master PISA de l'ENS. À travers la spécificité de Pompéi, il a permis de familiariser les étudiants aux techniques de la construction romaine. Suite à une initiation sur l'ensemble du site, des exercices d'analyse et de relevé ont été conduits dans la Villa de Diomède, portant sur les différents secteurs d'étude sélectionnés. Une conclusion de cette "spring school " a été présentée à l'occasion d'une journée organisée auprès de l'Institut français de Naples, le 21 mai 2014. 
Une mission complémentaire, réunissant les différents spécialistes et le photographe de la mission, Thomas Crognier, a été organisée du 3 au 20 novembre 2014.

4 Grâce à un élargissement de l'équipe scientifique et au soutien du Labex TransferS, la campagne 2014 a aussi permis d'affiner les méthodes utilisées, en particulier pour le système d'information géographique, les bases de données et le traitement de la documentation photographique.

\section{Un système de gestion de bases de données}

5 À l'occasion de la mission 2014, un système de gestion de bases de données a été échafaudé, comprenant deux outils réalisés avec le logiciel FileMaker :

1. Enregistrement informatique des unités stratigraphiques, avec une restructuration de la base préexistante OPUS (Outil Pour Unités Stratigraphiques construites).

2. Stockage, archivage et consultation de la documentation graphique (en particulier archives anciennes) et photographiques relative à la Villa de Diomède.

\section{Un Système d'Information Géographique pour l'étude de la villa}

6 C'est afin de cerner l'organisation du bâtiment, en exploitant au mieux les données de l'archéologie de la construction et les différentes sources d'archives anciennes disponibles, que la mission 2014 a vu la réalisation d'un plan vectoriel intégré dans un Système d'Information Géographique, où chaque élément architectural de la villa correspond à une entité géométrique géoréférencée (fig. 2). 
Fig. 2 - Vue 3D du plan des trois niveaux de la villa.

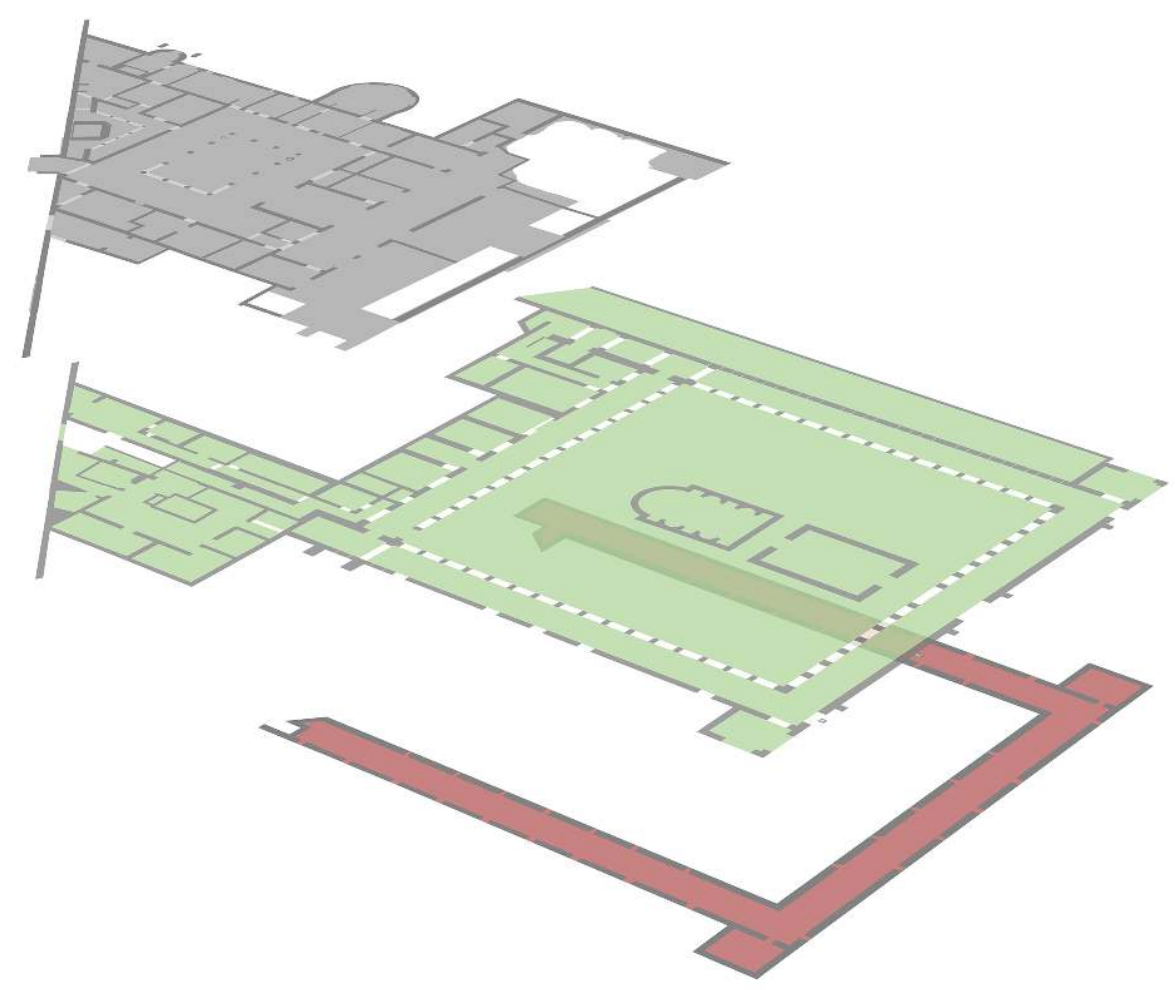

J. Cavero.

7 Il s'est agi de compiler les plans existants et de les repositionner dans le système de coordonnées géographiques Roma 1940 Gauss Boaga est. Nous avons utilisé le plan général des vestiges de Pompéi, probablement établi à partir de plusieurs sources dont des photographies aériennes, un plan géoréférencé comportant le réseau viaire du site ainsi que les points topographiques de référence implantés par la Surintendance en 2006 et un plan de la Villa de Diomède réalisé en 1997 dans le cadre d'une restauration architecturale pour la Surintendance.

8 Ce premier travail a servi de base aux deux stagiaires architectes, Stefano D'Arco et Gabriele Pepe, qui ont pu le corriger et le compléter et ainsi livrer un plan actualisé de la villa où chaque entité pourra être enrichie de multiples informations. C'est le cas des données renseignées dans la base OPUS-Pompéi ou de l'identification des différents chantiers de construction. L'objectif est aussi d'offrir à tous les membres du projet une base d'échange pour l'ensemble des données traitables en planimétrie 2D.

\section{Première réflexion sur les structures de la propriété}

L'analyse de l'évolution architecturale interne de la villa ne peut se faire sans une réflexion sur son insertion dans le tissu surburbain de Pompéi et le territoire, étude qui a été initiée cette année. En complément de l'analyse stratigraphique entreprise sur les façades du monument, l'objectif est de préciser sa relation avec la via delle Tombe et la nécropole adjacente (fig. 1). C'est la relation entre la villa et la nécropole au sud qui est apparue d'emblée la plus significative. En effet, si l'on observe sur toute la longueur de son tracé le mur de l'enclos funéraire qui jouxte la villa, on constate qu'il est parallèle à la 
voie, sauf dans sa dernière partie, à l'ouest, au contact avec la villa : là, le mur oblique fortement vers le nord. L'explication la plus simple de ce phénomène est de penser que les constructeurs ont évité d'englober dans l'enceinte funéraire l'abside du caldarium des thermes, pourvue d'une fenêtre (11) (fig. 3). Une interprétation possible est que le terrain sur lequel se situe l'enclos funéraire a été cédé par les propriétaires mêmes de la villa et que la zone où est établi l'enclos, probablement initialement un secteur de jardin, était - au moins partiellement - rattachée au même praedium que la villa. Plusieurs indices pourraient témoigner de cette association, comme les portes de communication entre l'intérieur de la villa et ce secteur méridional $(18,13)$ et les larges fenêtres aménagées, comme dans la pièce 17 ou le grand portique 63, même si certaines ont pu être obturées dans un dernier état. La relation entre l'enclos funéraire et la Villa de Diomède doit être aussi abordée en termes de circulation entre la via delle Tombe et le littoral et il s'agira, lors des prochaines campagnes, de préciser l'existence et le statut des différents passages conduisant de la voie à la mer. Prévues en 2015 grâce à l'appui d'un projet structurant PSL, des prospections géophysiques devraient permettre de préciser ces données et de mieux connaitre l'organisation des propriétés dans la zone sud et est de la via delle Tombe.

\section{Bilan provisoire sur l'identification des phases de construction}

10 À l'issue de cette campagne, nous présentons ici de premières hypothèses sur les phases de construction de la villa, qui demanderont bien évidemment à être vérifiées et précisées lors des prochaines missions. Sur le chantier de construction initial de la villa, nous n'avons pu recueillir que des indices très fragiles. En effet, si l'analyse des deux façades menée en 2014 avait pour objectif de préciser l'évolution des limites de la villa et de mettre en évidence d'éventuels témoignages de construction antérieurs à l'ouverture de la via delle Tombe, force est de constater que les résultats restent ténus et se limitent à trois points principaux. En premier lieu, le seul indice tangible d'extension de la villa à l'est est constitué par les restes d'un mur visible au niveau du trottoir, à l'est de la villa (fig. 3). 
Fig. 3 - Identification des phases de construction dans le secteur d'étude 2014, rez-de-chaussée.

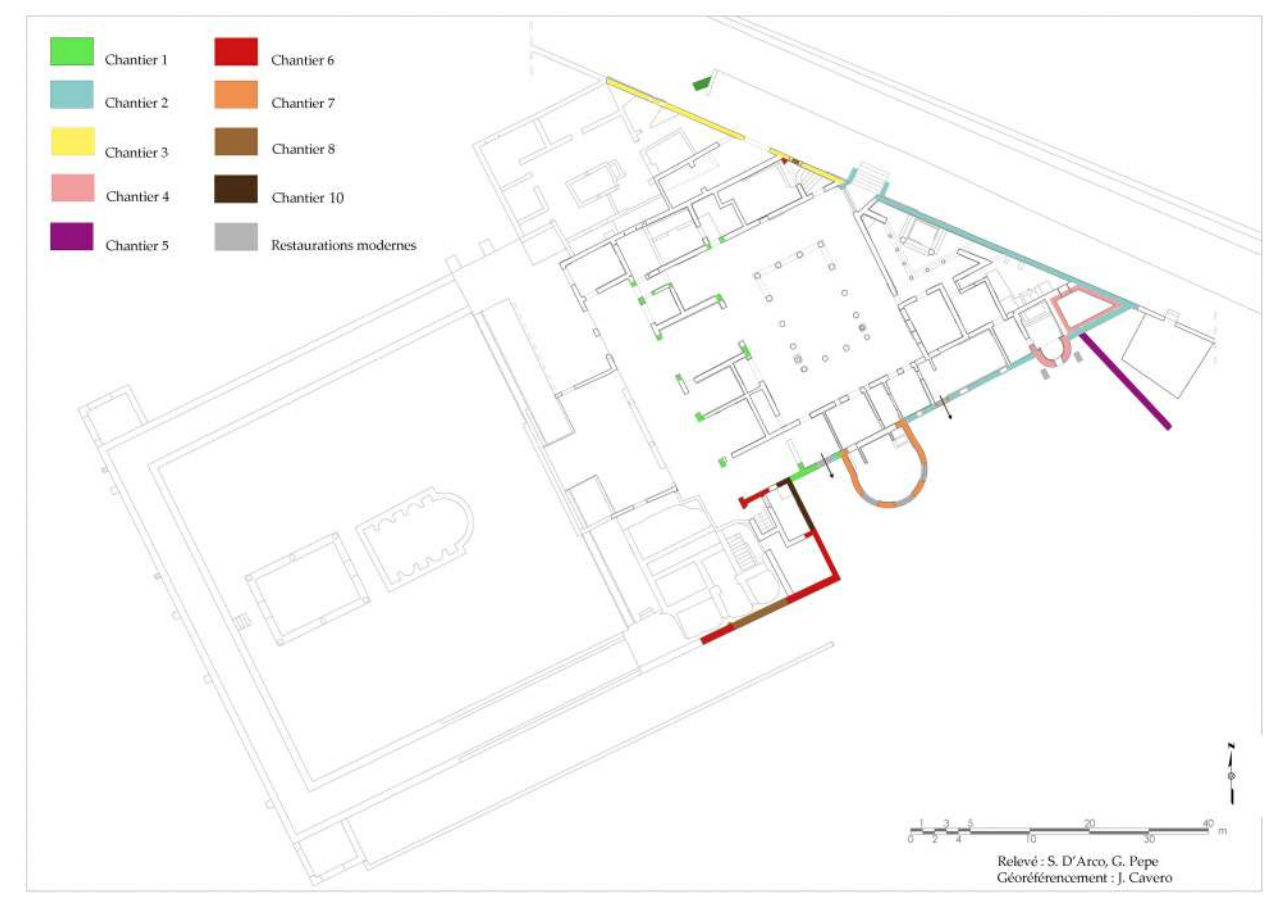

J. CAVERO, S. D'ARCo, G. PEPE, H. DEssales.

D'orientation est-ouest, conservé sur $70 \mathrm{~cm}$, il est aligné sur la trame de la villa, toutefois sans aucune continuité avec un de ses murs. Il présente un parement en opus reticulatum, à moellons de lave, seul le parement nord étant visible. Sa conservation est bien trop partielle pour que l'on puisse assurer qu'il était effectivement associé à un premier état de la villa, que la construction de l'axe viaire serait venue entailler. En second lieu, la trace d'un retour d'un angle de mur enduit (ou restes d'un pilastre d'angle) dans le secteur 100 n'a pas pu être véritablement éclaircie (US 100.001). En l'état des données, il reste difficile de croire qu'il s'agisse d'une première limite de la villa, ne se trouvant dans la continuité d'aucun mur interne au bâtiment. En troisième lieu, dans le secteur 104, une phase préexistante à l'aménagement unitaire de la façade sud a pu être mise en évidence. Il s'agit d'une porte assurant la communication entre la pièce 18 , dont elle occupe le centre du mur nord, et l'espace 31, devenu par la suite un espace de jardin, mais dont nous ne connaissons pas la configuration initiale ni le rapport au bâti préexistant. Les piédroits sont constitués de gros blocs de calcaire du Sarno, associés à une élévation d' opus incertum (US 104.013 et 104.017) et surmontés d'un arc, dont le négatif est encore clairement visible (fig. 5).

Le montant ouest en a ensuite été retaillé (US 104.018) et l'arc partiellement comblé (US 104.015), pour l'aménagement d'une fenêtre. Dans son premier état, l'ouverture est large de 1,74 m, haute de plus de 2,28 m (la partie basse n'étant pas visible). L'enduit qui couvrait les montants et l'arc est encore visible (US 104.11 et 104.16). Cette phase de construction, qui correspond à l'implantation initiale de la villa, peut être identifiée dans d'autres secteurs du bâtiment, avec des piédroits en blocs de calcaire du Sarno, assemblés à sec et associés à un opus incertum majoritairement constitué de moellons de lave, et tout particulièrement sur l'alignement ouest de toutes les pièces de réception donnant sur la loggia $(19,22,35,36,34$ et 18). Une cartographie et une typologie détaillée de ces 
éléments est prévue pour la campagne $2015^{2}$. Ce type de technique de construction se diffuse largement dans les demeures de l'aristocratie samnite au cours du II ${ }^{\mathrm{e}}$ siècle av. J.c. L'examen de la stratigraphie de la façade sud indique que cette porte, comme le premier agencement des espaces ouvrant sur la loggia, constitue la première phase de construction identifiée dans l'évolution du bâtiment.

En dehors de ces aménagements, limités au secteur central de la villa, l'édification des façades semble s'intégrer dans un projet relativement unitaire, à mettre en rapport avec la création de la via delle Tombe, aucun élément préexistant ne pouvant être mis en lumière. Ces conclusions rejoignent celles avancées par D. Esposito sur la première phase de la Villa des Mystères, dont l'examen du décor ne permet pas d'avancer une datation antérieure à la fin du $\mathrm{II}^{\mathrm{e}}$ ou début du $\mathrm{I}^{\mathrm{er}}$ siècle av. J.-C. ${ }^{3}$.

Sans entrer dans le détail des phases ponctuelles de réfection mises en évidence sur les façades (réfections partielles des élévations, des ouvertures, reprises en sous-œuvre, et détaillées dans les rapports par secteurs), nous rassemblons ici les grandes phases de chantier de construction pouvant être identifiées (fig. 4 et 5).

Fig. 4 - Identification des phases de construction dans le secteur d'étude 2014, rez-de-jardin.

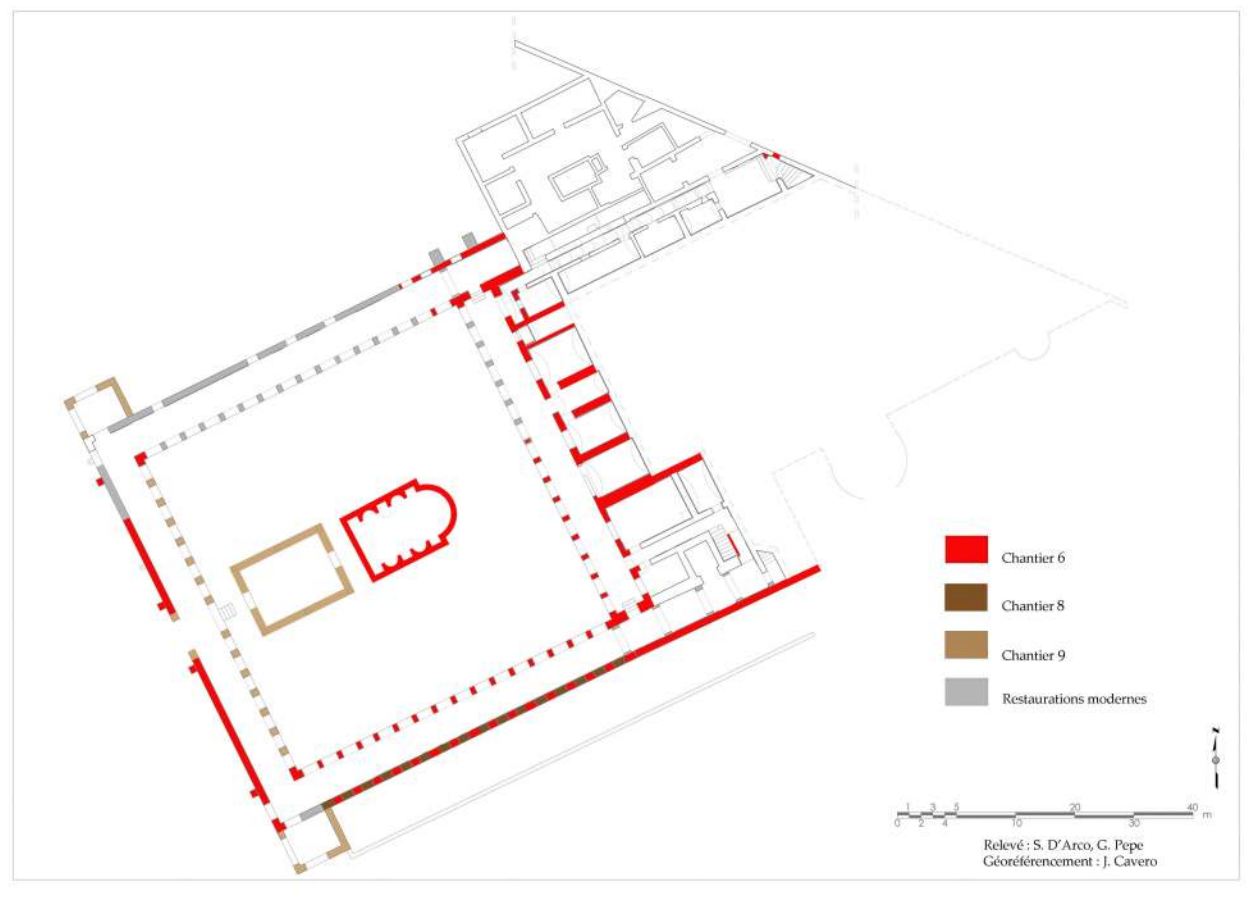

J. CAVero, S. D'ARCo, G. PEPE, H. Dessales. 
Fig. 5 - Secteur 104, mur nord, parement sud : identification des phases de construction.
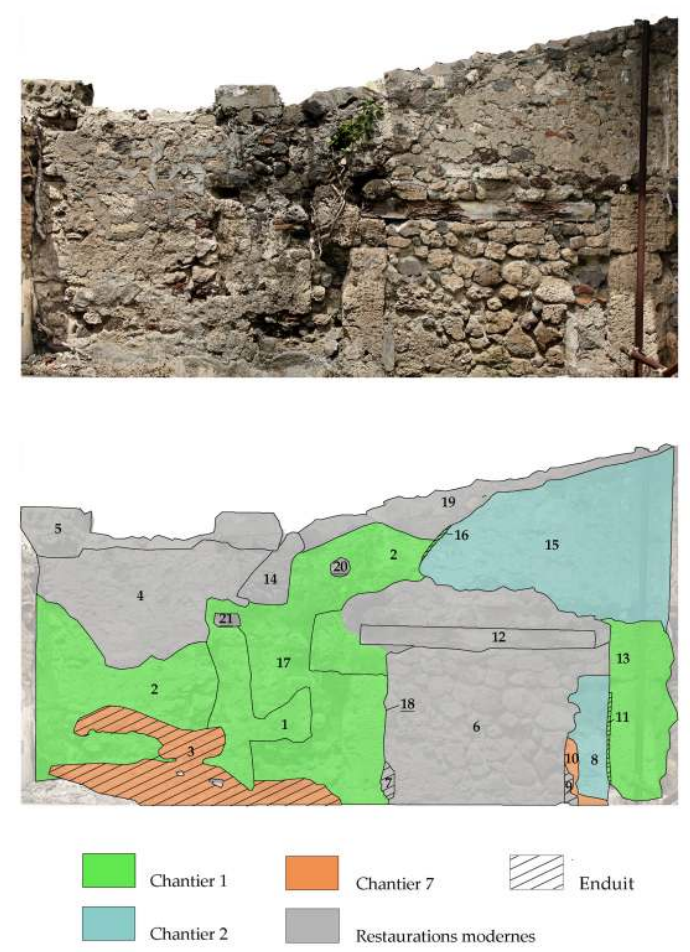

Photographie T. Crognier ; Étude A. Péron, S. lavarone ; DAO H. Dessales.

1. Corps central de la villa avec piliers en blocs de calcaire. Si la limite du bâtiment demeure inconnue à l'est, c'est une loggia à portique, dont les colonnes ont ensuite été englobées dans les maçonneries lors de la phase d'extension sur le jardin, qui assurait vraisemblablement la limite ouest au niveau du rez-de-chaussée.

2. Monumentalisation de la façade est, avec escalier d'entrée et secteur septentrional. De façon contemporaine, réfection de la façade sud et transformation de la porte de la pièce 18 en fenêtre; une communication avec le jardin au sud est alors assurée par la porte aménagée dans la pièce 12.

3. Réaménagement du secteur septentrional de la façade est, en rapport avec le secteur de service.

4. Aménagement de l'espace thermal et insertion de l'abside du caldarium 11 dans la façade sud.

5. Construction du mur d'enceinte des tombes adjacentes au sud, nécessairement postérieur à l'abside thermale.

6. Aménagement (avant le tremblement de terre de 63 ap. J.-C.) de la terrasse 44 et du grand portique et des espaces du rez-de-jardin, probablement dans les années 50 ap. J.-C.

7. Construction de la pièce absidée et restructuration des systèmes de circulation dans le secteur sud-ouest de la villa, avec fermeture de la porte vers le jardin 31.

8. Restructuration et bouchage des fenêtres du grand portique ouvrant sur le jardin, ainsi que d'une partie de l'élévation du portique, probablement suite au tremblement de terre de 63 ap. J.-C.

9. Réfection du portique ouest sur le jardin et des tours d'angle, suite à un autre épisode sismique? 
10. Interventions diverses (réfections de murs, bouchages de baies), dont la datation relative doit encore être précisée.

L'attention s'est concentrée sur les systèmes de circulation entre la villa et l'espace qui la borde au sud et dont la fonction reste à déterminer (jardin? accès vers la mer en contrebas?). Les phases d'évolution du grand péristyle en contrebas de la villa ont ainsi été ou être précisées. Plusieurs points avaient déjà été avancés dans le rapport 2013 à ce sujet. La première phase de construction du portique, associée à des peintures de Quatrième Style, se caractérise par l'usage de moellons en tuf gris de Nocera et en tuf jaune, régulièrement taillés en forme de trapèze ou de parallélogrammes, dans les murs comme dans les piliers (fig. 6).

Fig. 6 - Portique 63, détail mur est, parement ouest $\left(1 \mathrm{~m}^{2}\right)$, avec moellons taillés en trapèzes ou parallélogrammes.

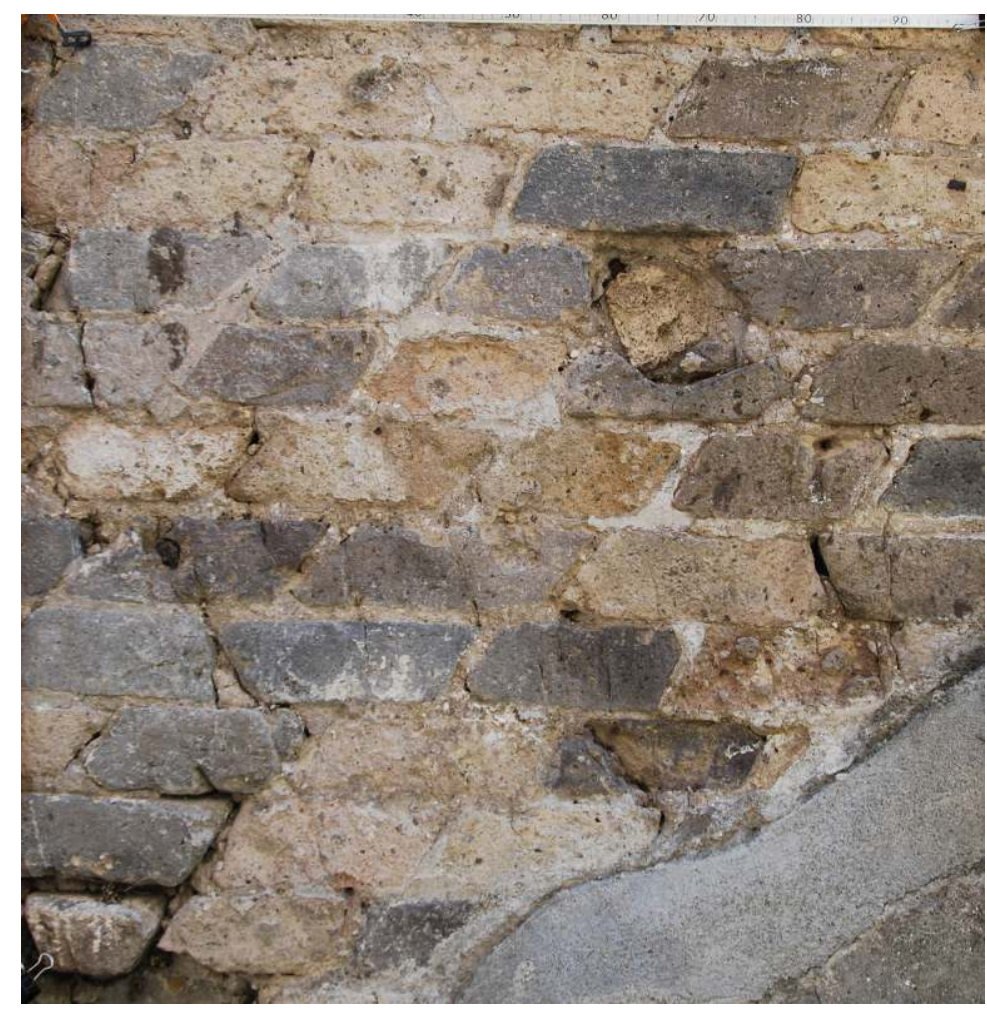

H. Dessales.

Dans cette première phase, une série de seize fenêtres régulières sont organisées dans le mur sud, ouvrant sur l'espace 75 (fig. 4). La réfection moderne du mur nord ne permet pas d'en restituer la symétrie, mais la présence d'une ouverture bouchée à l'extrémité de la galerie nord, dans l'espace 68, ouvrant sur l'espace 88, pourrait être un indice en ce sens. Dans une phase successive, probablement suite au tremblement de terre de 63 ap. J.-C., les ouvertures sont systématiquement modifiées par différentes opérations. La configuration des moellons dans certaines des plates-bandes laisse supposer un écroulement partiel, qui a imposé d'ôter le linteau de bois (dont la présence initiale était délimitée par des fragments de TCA), de restructurer la maçonnerie des plates-bandes avec les moellons préexistants et les fragments de TCA et de boucher l'ouverture par une maçonnerie grossière de moellons de tuf (fig. 7). 


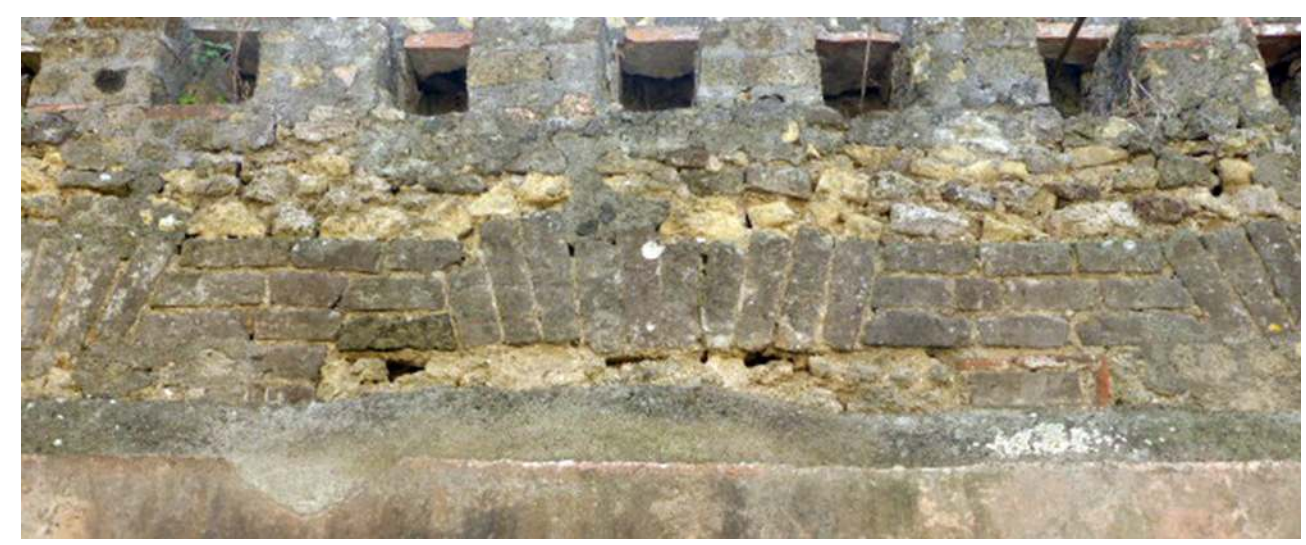

Bouchage d'une ouverture et de la plate-bande, avec réfection du linteau.

H. Dessales.

Enfin, dans un dernier état, sont construites les deux tours d'angle (fig. 5). Il ne peut s'agir d'une phase successive de chantier, dans la mesure où la tour sud vient clairement s'adosser au bouchage de la fenêtre du mur sud. Leur élévation se caractérise par l'usage de fragments de TCA et de moellons rectangulaires en tuf de Nocera, disposés en assises régulières. La même technique est utilisée dans la construction de l'ouverture sur le mur ouest, en direction de la mer, dans une partie reconstruite de ce dernier, dans les piliers du portique ouest et dans la base du triclinium d'été. Il semblerait qu'il s'agisse de la dernière grande phase de monumentalisation de la villa, probablement suite à une destruction dans son secteur ouest.

19 La mise en évidence de ces différents chantiers révèle les capacités de réaction des propriétaires et, tout particulièrement, le dynamisme des constructions dans les dernières années qui ont précédé l'éruption finale de 79.

\section{Étude des revêtements muraux}

20 À la suite de l'état des lieux réalisé lors de la mission 2013, nous avons cette année entamé l'analyse des revêtements muraux pièce par pièce, mis en perspective avec les sols et l'architecture. Notre attention s'est portée sur le secteur des bains, délimité au nord et au nord-est par les murs de façade dont les parements extérieurs étaient étudiés du point de vue des techniques de construction. L'observation des vestiges conservés en place a été confrontée à la riche documentation d'archives dont on dispose pour ce secteur, afin de comprendre et de restituer au mieux le décor des voûtes et des parois.

21 Le péristyle 4-5, la pièce 10 et le caldarium 11 ont tous trois livré plusieurs phases de revêtements pariétaux. Les décors conservés appartiennent presque tous au Quatrième Style pompéien et les diverses réfections observées peuvent donc être situées dans les dernières décennies de la vie de la villa. La seule trace identifiable d'une phase antérieure est pour l'instant la voûte stuquée et peinte de l'abside du caldarium 11, dont il nous reste à préciser la datation (fig. 8). 
Fig. 8 - Caldarium 11, mur sud, parement nord.

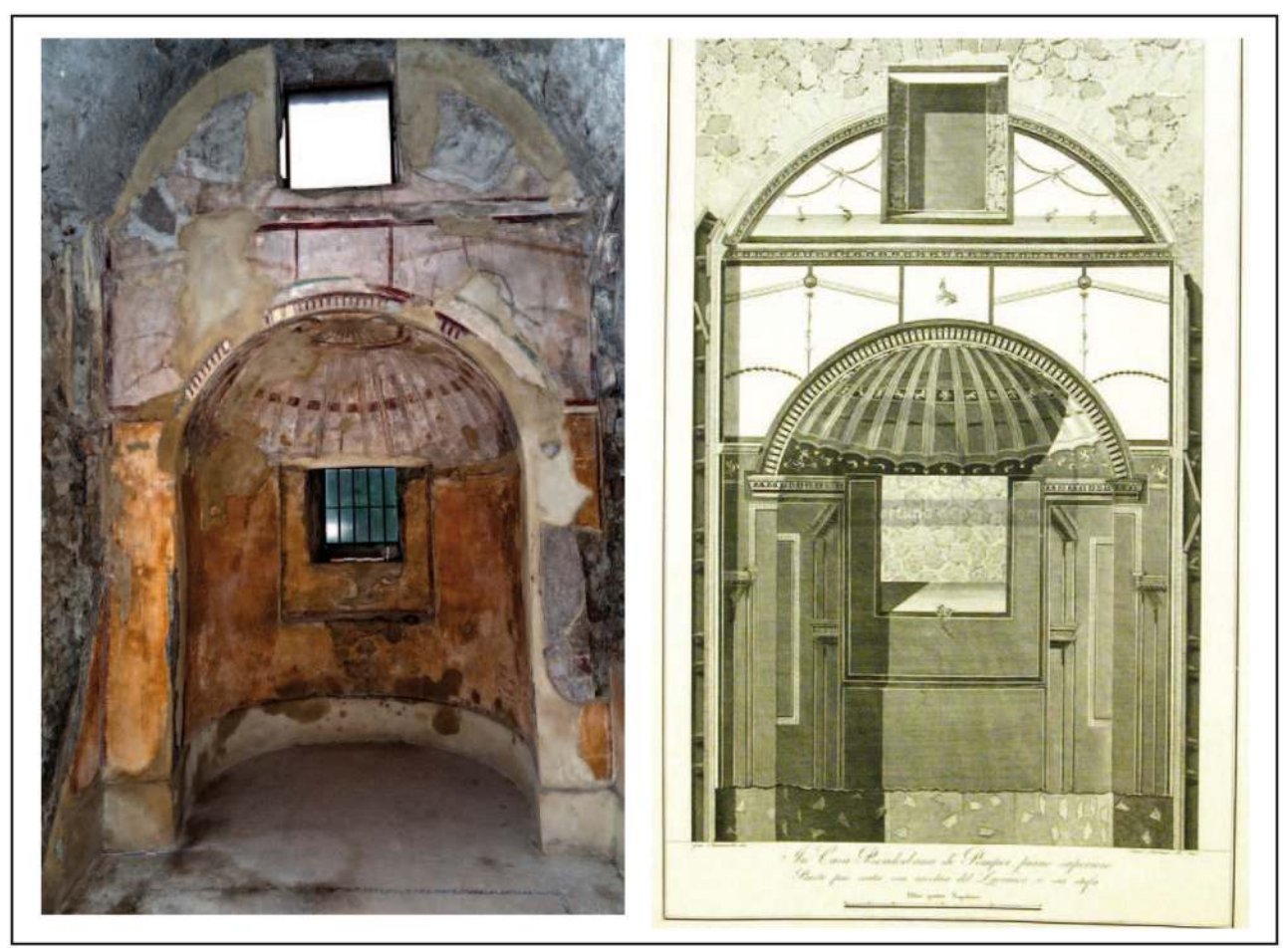

Photographie de l'état en 2014 (T. Crognier) et relevé à l'issue des fouilles, 1798.

Cliché T. Crognier ; G. Chianterelli in Gli Ornati... 1796-1808, vol. 2, 63.

On note, dans ce secteur, une richesse ornementale croissante entre les parois de la galerie 5 et les pièces 9,10 et 11 , le caldarium, pour autant qu'on puisse en juger, ayant le décor le plus raffiné. La pièce 12 présente également plusieurs phases de revêtements pariétaux, cependant difficiles à dater car pas ou peu décorés. Ils témoignent en revanche d'une phase où l'on pouvait accéder au jardin depuis cette pièce, puisqu'ils ont conservé le négatif de marches installées devant la grande fenêtre du mur sud (fig. 3).

L'étude du secteur des bains sera poursuivie au cours de la prochaine mission et les peintures attentivement comparées à celles des pièces du rez-de-jardin, dont les décors appartiennent également pour la plupart au Quatrième Style, mais avec des différences stylistiques notables qu'il faudra interpréter.

\section{Étude des pavements}

Le travail sur le terrain a été privilégié lors de cette campagne, suite à l'examen des pavements des musées de Naples et de Portici déjà bien avancé au cours de la campagne 2013. Des études ponctuelles ont été menées au rez-de-chaussée afin de vérifier les caractéristiques et l'étendue du revêtement en cocciopesto du péristyle 2 et le mettre en relation avec les données stratigraphiques des pièces attenantes et surtout du portiqueterrasse 41. La surface du cocciopesto s'arrête au niveau des seuils des pièces ouvrant sur le péristyle sauf pour l'espace 23 où subsiste la partie inférieure du sol, trop détérioré et recouvert d'herbe pour que son décor soit reconnaissable. Dans cette zone de transition entre les pièces 23 et 33 la décoration du sol change. C'est la même base de mortier qui est 
utilisée dans le péristyle 2 et le portique-terrasse 41, mais sans semis de tesselles ou de croisettes.

À partir des observations faites dans les espaces du rez-de-chaussée, un premier schéma du programme décoratif peut être proposé (fig. 9). Il s'appuie sur les vestiges encore in situ (pièces 9 à 11 , péristyle 2 , atrium 4 et 5 , pièces 34 à 36 et 40 , portique 41 ) ainsi que sur les descriptions des journaux de fouilles (à propos des pièces 14, 17 et 22).

Fig. 9 - Rez-de-chaussée, état de l'étude des schémas décoratif proposés, avec quelques témoins.

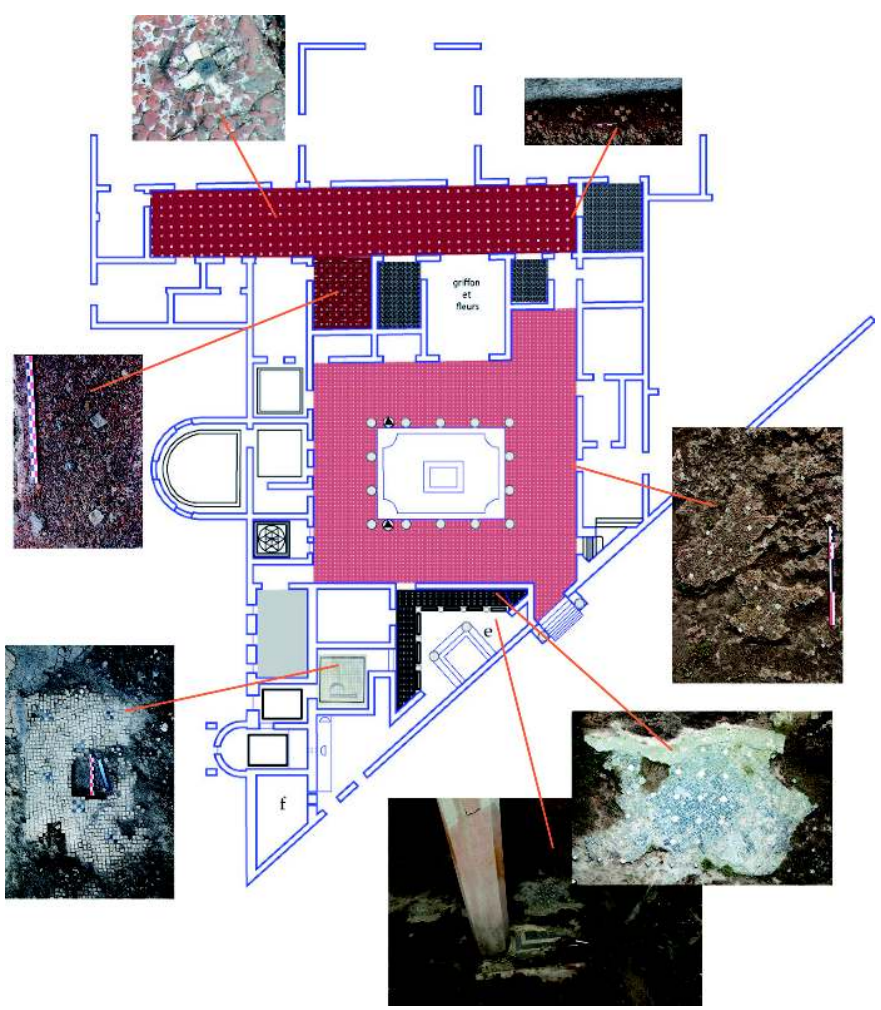

F. Marchand-Beaulieu.

Après nettoyage du pavement de la pièce 49 , nous avons découvert que les bordures noires sur fond blanc passaient sous le mur ouest de la pièce et se retrouvaient dans la berme d'un sondage effectué de l'autre côté du mur. Les pièces 49 et 53 (du moins dans sa partie est) n'en formaient donc qu'une seule dans une phase d'occupation antérieure. Cependant, la spoliation de la mosaïque de la pièce 53 prive de précieux indices quant aux dimensions et aux modes d'accès de cette grande salle. Il est difficile en l'état actuel de la recherche de déterminer la chronologie relative de ces modifications architecturales et décoratives.

\section{Étude des graffitis historiques}

$\mathrm{Au}$ cours de cette seconde campagne, l'étude s'est concentrée sur les pièces d'apparat du rez-de-jardin $(54,55,56$ et 57$)$, ainsi que sur l'extrémité orientale de l'aile sud du portique 69. Les 52 dates indiquées dans les graffitis témoignent d'une même tendance que celle notée dans le caldarium (11), avec une forte fréquentation dans la première moitié du XIX ${ }^{\mathrm{e}}$ siècle, qui diminue ensuite pour totalement disparaître dans la seconde moitié du $\mathrm{XX}^{\mathrm{e}}$ 
siècle, la fréquentation de ces pièces culminant dans les années 1820-1830. Les 137 noms clairement identifiés permettent d'établir l'origine des voyageurs, les Italiens restant largement majoritaires (près de la moitié), suivis d'un groupe important d'anglo-saxons (47\% des visiteurs étrangers). Quelques noms de personnages se détachent, notamment l'écrivain anglais Henry Swinburne, qui évoque dans son récit de voyage la Villa di Diomede $^{4}$, le philosophe Johann Gottfried von Herder, voyageant en Italie en 1788 et l'architecte Paul-Emile Bonnet, prix de Rome en 1854.

Prévue en 2015, une collaboration avec le C2RMF soutenue par un projet structurant PSL, devrait permettre, grâce à différentes techniques d'imagerie scientifique, de révéler les traces d'inscription au carbone, invisibles à l'œil nu et d'élargir considérablement le corpus

\section{Perspectives 2015}

Afin de préciser les premiers phasages obtenus à l'issue de cette campagne sur les façades de la villa, il conviendra, lors de la prochaine campagne, d'approfondir l'enquête dans les secteurs internes correspondants, en particulier dans les pièces articulant péristyle, cuisine et bloc thermal (fig. 1, pièces 4 à 12). En effet, si l'étude des décors en a déjà pu être engagée, une analyse stratigraphique systématique doit être conduite sur les murs du secteur. Dans ce cadre, sur le même principe qu'en 2014, un stage de formation à l'archéologie de la construction sera proposé à une dizaine d'étudiants archéologues et architectes.

Par ailleurs, dans la continuité des observations menées en 2014, il s'agira de préciser le rôle des impacts sismiques dans les différents chantiers de reconstruction de la villa. Une collaboration a été engagée sur cet aspect avec les départements d'ingénierie de l'Università degli Studi di Padova et l'Università degli Studi di Napoli Federico II, sous la direction de Claudio Modena et Andrea Prota, afin de préciser le comportement structurel du bâtiment et sa résistance aux tremblements de terre. Plus généralement, cette thématique s'inscrira dans un programme de recherche de plus grande ampleur sur Pompéi, soutenu par l'ANR à partir de $2015^{5}$. Il aura pour objectif d'apporter un nouvel éclairage sur le développement d'une architecture du risque et de l'urgence à l'époque romaine. Sur ce laboratoire exceptionnel que constitue Pompéi, un des modules principaux sera consacré à l'analyse de la Villa de Diomède, à travers la mise en évidence des opérations de réfection et des éventuelles innovations techniques. Au-delà de cette étude de cas, une plus large réflexion sera proposée sur la mémoire du risque en Campanie.

\section{BIBLIOGRAPHIE}

Gli Ornati... 1796-1808 = G. Chiantarelli, in Gli Ornati delle pareti ed i pavimenti delle stanze dell'antica Pompei incisi in rame, Naples, 1796-1808, vol. 2, 63. 
Esposito 2007 = D. Esposito, Pompei, Silla e la Villa dei misteri, dans B. Perrier (éd.), Villas, maisons, sanctuaires et tombeaux tardo-républicains : découvertes et relectures récentes [Actes du colloque international de Saint-Romain-en-Gal en l'honneur d'Anna Gallina Zevi, 8-10 fév. 2007], Rome, 2007, p. 441-465.

Kockel - Weber 1983 = V. Kockel, B. Weber, Die Villa delle colonne a mosaico, dans MDAI(R), 90, 1983, p. 51-89.

Swinburne 1783-1785 = H. Swinburne, Travels in the Two Sicilies, in the years 1777, 1778 and 1780, Londres, 1783-1785.

\section{NOTES}

1. http://cefr.revues.org/1121. Voir également le site web du projet (réalisation Thomas Crognier) : www.villadiomedeproject.org/

2. Pour une première localisation de ces éléments dans la villa, voir Kockel - Weber 1983, p. 67, fig. 5. Cette typologie demande toutefois à être précisée, car, comme on le note dans le cas de la Villa di Diomede, il convient de distinguer les piédroits aménagés de façon originelle avec des blocs de calcaire du Sarno et ceux ayant recours à des blocs de remploi, dans des états successifs de la villa. Voir par exemple le cas des piédroits de la pièce 14, présentée dans le rapport 2013 et constituée de blocs de remploi liés au mortier.

3. Esposito 2007, p. 454.

4. Swinburne 1783-1785, vol. 3, p. 152-155.

5. ANR 14-CE 31-005 / RECAP (Reconstruire après un séisme. Expériences antiques et innovations à Pompéi), coordination scientifique H. Dessales, AOROC (UMR 8546 ENS-CNRS), en collaboration avec l'Inria, l'IPGP et le Centre Jean Bérard (USR 3133 EFR-CNRS). Ce projet est fondé sur une approche fortement interdisciplinaire qui sollicite archéologie de la construction, sociologie, informatique, ingénierie structurelle et géologie.

\section{INDEX}

Index géographique : Pompéi

institutions École normale supérieure (UMR 8546 et 8548) / PSL Research University, CNRS (Soutien à la mobilité internationale), Labex TransferS, Centre Jean Bérard, Institut National de Recherche en Informatique et Automatique, Centre de recherche commun Inria-Microsoft Research, Institut Universitaire de France, Ministère des Affaires Étrangères, Soprintendenza speciale per i beni archeologici di Napoli e Pompei

Mots-clés : villa romaine, architecture, décor, archives, relevé informatique, voyageur européen 


\section{AUTEURS}

\section{HÉLÈNE DESSALES}

ENS, AOROC (UMR 8546), PSL Research University, Institut Universitaire de

France - Helene.Dessales[at]ens.fr

\section{JEAN PONCE}

WILLOW, Département d'informatique de l'ENS (UMR 8548), PSL Research University, Institut Universitaire de France - Jean.Ponce[at]ens.fr

\section{MATHILDE CARRIVE}

École française de Rome

JULIEN CAVERO

Labex Transfers

JULIEN DUBOULOZ

Université Aix-Marseille, Centre Paul Albert-Février (UMR 7297)

ÉLOÏSE LETELLIER

École française de Rome

FRÉDÉRIQUE MARCHAND- BEAULIEU

CNRS, AOROC (UMR 8546)

\section{FLORENCE MONIER}

CNRS, AOROC (UMR 8546)

AMBRE PÉRON

ENS

AGNÈS TRICOCHE

Labex Transfers

YVES UBELMANN

Iconem / IRAA, USR 3155 